\title{
A influência do género dos gestores das microempresas na utilidade atribuída à contabilidade
}

por Henrique Gouveia, Joaquim Fernandes, Cristina Gonçalves e Gabriela Gonçalves

RESUMO: A informação contabilística tem como função fornecer dados necessários à tomada de decisão dos gestores. As perspetivas de género defendem existir diferenças nos valores e atitudes de risco. Neste estudo definiram-se dois objetivos: avaliar as diferenças de género no recurso à informação contabilística e observar a utilidade atribuída à informação contabilística e a sua importância para a tomada de decisão dos gestores em função do género. Com base em 609 gestores de microempresas selecionados por conveniência, delineou-se um design bifatorial de 2 (género: homens versus mulheres) X 2 (utilizador: sim versus não) com recurso a um inquérito online. Os resultados não apresentam diferenças significativas de género entre os gestores que utilizam a contabilidade como fonte de informação. No entanto, de entre os gestores não utilizadores da informação contabilística, as mulheres reconhecem de forma diferente a utilidade dessa informação.

Palavras-chave: Informação Contabilística; Género; Microempresas; Tomada de Decisão

\section{La influencia del género de los gestores de las microempresas en la utilidad atribuida a la contabilidad}

RESUMEN: La información contable tiene como función proporcionar los datos necesarios para la toma de decisión de los gestores. Las perspectivas de género defienden la existencia de diferencias en los valores y actitudes de riesgo. En este estudio se definieron dos objetivos: evaluar las diferencias de género en el recurso a la información contable y observar la utilidad atribuida a la información contable y su importancia para la toma de decisión de los gestores en función del género. Con base en 609 gestores de microempresas seleccionados por conveniencia, se delineó un diseño bifactorial de 2 (género: hombres versus mujeres) X 2 (usuario: sí versus no) con un estudio online. Los resultados no presentan diferencias significativas de género entre los gestores que utilizan la contabilidad como fuente de información. Sin embargo, entre los gestores no usuarios de la información contable, las mujeres reconocen de forma diferente la utilidad de dicha información.

Palabras clave: Información Contable; Género; Microempresas; Toma de Decisiones 


\section{The influence of the manager's gender in the utility assigned to accounting}

ABSTRACT: The accounting information has the function of providing data and information necessary for the manager's decision-making. Gender perspectives suggest that there are differences in risk values and attitudes. The first objective of this article was to evaluate gender differences in the use of accounting information and the second objective was to observe the usefulness attributed to accounting information and its importance for the managers decision-making according the gender. Based on 609 managers of microenterprises selected by convenience, a two-factor design was delineated from 2 (gender: men versus women) X 2 (user: yes versus no) using an online survey. The results obtained do not present significant gender differences among managers who use accounting as a source of information. However, among managers who do not use accounting information, women recognize differently the usefulness of this information.

Key words: Accounting Information; Gender; Micro-Enterprise; Decision Making

Henrique Gouveia

henrique.mng@hotmail.com

Mestre em Contabilidade, Universidade do Algarve, Faculdade de Economia. Contabilista certificado, autor de diversos artigos científicos na área. Universidade do Algarve, Campus da Penha 8005-139 Faro.

Maestría en Contabilidad, Universidad del Algarve, Facultad de Economía. Contable certificado, autor de diversos artículos científicos en el área. Universidad del Algarve, Campus da Penha 8005-139 Faro.

Master in Accounting, University of Algarve, Faculty of Economics. Certified accountant, author of several research papers. University of Algarve, Campus da Penha, 8005-139 Faro.

Joaquim Sant'ana Fernandes

jsfer@ualg.pt

Doutorado em Gestão Empresarial. Professor na Universidade do Algarve, na área da Contabilidade. Autor de livros e diversos artigos científicos na área. Membro do Centro de Investigação sobre Espaço e Organizações, Universidade do Algarve, Campus da Penha, 8005-139 Faro.

Doctorado en Gestión Empresarial. Profesor en la Universidad del Algarve, en el área de Contabilidad. Autor de libros y diversos artículos científicos en el área. Miembro del Centro de Investigación sobre Espacio y Organizaciones, Universidad del Algarve, Campus da Penha, 8005-139 Faro.

PhD in Business Management, Professor of accounting in University of Algarve. Author of an accounting book and several research papers. Member of the Research Centre for Spatial and Organizational Dynamics, University of Algarve, Campus da Penha, 8005-139 Faro.

Recebido em novembro de 2015 e aceite em setembro de 2017 Recibido en noviembre de 2015 y aceptado en septiembre de 2017 Received in November 2015 and accepted in September 2017
Cristina Gonçalves

cjesus@ualg.pt

Mestre em Ciências Económicas e Empresariais. Professora na

Universidade do Algarve, na área da Contabilidade. Autora de diversos livros e artigos científicos na área. Membro do Centro de Investigação sobre Espaço e Organizações, Universidade do Algarve, Campus da Penha, 8005-139 Faro.

Maestría en Ciencias Económicas y Empresariales. Profesora en la Universidad del Algarve, en el área de Contabilidad. Autora de diversos libros y artículos científicos en el área. Miembro del Centro de Investigación sobre Espacio y Organizaciones, Universidad del Algarve, Campus da Penha, 8005-139 Faro. Master in Economic and Managerial Sciences and Professor of accounting in University of Algarve. Author of several research papers and books about accounting and professional ethics. Member of the Research Centre for Spatial and Organizational Dynamics, University of Algarve, Campus da Penha, 8005-139 Faro.

Gabriela Gonçalves

ggoncalves@ualg.pt

Doutorada em Ciências Psicológicas. Professora Auxiliar na Universidade do Algarve na área de Gestão de Recursos Humanos e Psicologia Social e das Organizações. Membro do Research Centre for Spatial and Organizational Dynamics, Universidade do Algarve, Campus de Gambelas, 8005-139 Faro.

Doctorado en Ciencias Psicológicas. Profesora Auxiliar en la Universidad del Algarve en el área de Gestión de Recursos Humanos y Psicología Social y de las Organizaciones. Miembro del Research Centre for Spatial and Organizational Dynamics, Universidad del Algarve, Campus de Gambelas, 8005-139 Faro. $\mathrm{PhD}$ in Psychological Science. Assistant Professor of Human Resources and Social and Organizational Psychology at the University of Algarve. Member of Research Centre for Spatial and Organizational Dynamics, University of Algarve, Campus de Gambelas, 8005-139 Faro. 
Segundo a Informa D\&B (2012), observa-se uma minoria feminina nos cargos de gestão (30,5\%), sendo nas microempresas (ME) que se verifica uma maior participação nesses cargos $(33,1 \%)$ e também nas empresas mais jovens onde assumem maior relevância. Já de acordo com o Instituto Nacional de Estatística (INE, 2013), 5,2\% do universo das mulheres empregadas em 2011 exerciam um cargo dirigente, apresentando um perfil mais jovem e mais qualificado do que o dos homens. Segundo o European Institute for Gender Equality (2013), Portugal, em 2010, estava entre os países com menor igualdade, com um índice de igualdade de género de 41,3, quando a média da União Europeia se situa nos 54 (em 100).

Os gestores sustentam os seus processos de decisão num conjunto de «ferramentas $\gg$. A informação contabilística (IC), entendida como toda a informação gerada com base em registos, tanto de natureza financeira, como económica, numa perspetiva histórica ou prospetiva, independentemente de a mesma ser obrigatória ou voluntária, é uma dessas «ferramentas» (ver Chen, Ding e Xu, 2014).

Não obstante as diferenças quantitativas existentes entre os géneros quanto à presença na gestão das empresas, diversos estudos têm analisado em que medida as diferenças entre estilos de gestão e processos de decisão podem ser explicados pelos perfis dos gestores (ver Rüzbar e Kurt, 2013). Neste contexto, o efeito das variáveis biográficas, nomeadamente o género, no processo de tomada de decisão tem sido um foco de interesse (ex., Delaney et al., 2015). Os resultados nem sempre são consensuais ou conclusivos sobre esse efeito (ex., Baiocco, Laghi e D’ Allessio, 2009; ver Spicer e Sadler-Smith, 2005).

Por um lado, alguns estudos apontam para uma atitude mais cautelosa, menos agressiva e por procura de apoio nas tomadas de decisão (ex., Harris, Jenkins e Glaser, 2006) por parte das mulheres em cargos de gestão. Por outro, Francis et al. (2014) afirmam que os responsáveis financeiros femininos são mais adversos ao risco. Ainda a este propósito, Filho, Bruni e Sampaio (2012) observaram a ocorrência de heurísticas em decisões orçamentais, sendo que a variável género apresentou uma relação significativa.

Estes e outros estudos apontam para a possibilidade de diferenças de género na importância e utilidade percebida da IC e da sua utilização na tomada de decisão dos gestores. Defende-se que a utilidade da IC, para a tomada de decisão, é percecionada de forma diferenciada pelo género do gestor.

Considerando a ampla aplicação que a IC tem para o aperfeiçoamento de produtos e processos das empresas, mas a pouca informação sobre a presença de gestoras, a aplicação nos conteúdos do ensino em contabilidade e a realidade portuguesa (Ferreira, 2013) desenvolveu-se um estudo que visa avaliar a utilidade percebida e a importância que cada género atribui à IC. Atendendo que nem sempre há consistência entre atitude e comportamentos (ver Ajzen e Fishbein, 1980), as variáveis foram analisadas em decisores que usam e não usam a IC com base num estudo bifatorial de 2 (género: homens vs. mulheres) X 2 (utilizador: sim vs. não). Definiu-se como objeto de investigação as ME portu-
Estes e outros estudos apontam para a possibilidade de diferenças de género na importância e utilidade percebida da informação contabilística e da sua utilização na tomada de decisão dos gestores. 
guesas [Comissão Europeia (CE), 2003], sob a forma de sociedade. Segundo o INE (2013), para dados de 2011, num universo de 300923 sociedades não financeiras, 254764 são ME, representando cerca de $85 \%$ das sociedades, razão pela qual se optou por estas entidades.

\section{Revisão da literatura}

O processo de tomada de decisão inicia-se com a recolha e análise da informação de suporte, pelo que existe uma relação de causa-efeito entre a informação sobre a qual se decidiu e a decisão (Davenport, 2009). A qualidade da avaliação que se faz das informações disponíveis determina o sucesso da decisão (ver Mala e Chand, 2015).

A informação contabilística surge no contexto do processo de tomada de decisões com a função de fornecer dados e elementos que, em determinada altura, serão necessários para a resolução dos problemas (Caplan, 1966) de natureza estratégica e/ou operacional (Davidson e Trueblood, 1961). Vários estudos (ver Mala e Chand, 2015), apoiados em diferentes metodologias, confirmam a importância da qualidade da informação contabilística e da sua avaliação para o sucesso das tomadas de decisão (estratégica e operacional) e para os investidores (ex., Chen, et al., 2014; Kang e Stulz, 1997; Portes e Rey, 2005). Rossi e Volpin (2004) compararam o volume de fusões e aquisições transfronteiriças e observaram que os países com melhores informações contabilísticas apresentam valores significativamente superiores aos outros países.

Também o estudo de Martin (1971) evidencia claramente a relevância dos dados dos relatos contabilísticos anuais na área das decisões estratégicas, nomeadamente nas decisões de investimento. Esta ideia é reforçada pelo modelo de Zhang (2000) ao reconhecer que os dados contabilísticos contêm informação útil para guiar as decisões de investimento, sendo que este investimento constitui a base da criação de valor empresarial. Okoh e Uzoka (2012) realizaram uma investigação sobre o papel da informação contabilística na sobrevivência das pequenas empresas na Nigéria em que observaram uma relação relevante entre a informação contabilística e a sobrevivência das empresas. O trabalho de Lucena, Vasconcelos e Marcelino (2011) identificou quais as informações contabilísticas utilizadas no processo de tomada de decisão por parte dos gestores das pequenas e microempresas de uma localidade brasileira. Concluíram que as empresas são influenciadas pelo uso dos relatórios contabilísticos, mas que existe a necessidade de uma melhor gestão da informação contabilística para a tomada de decisão.

Em Portugal, destaca-se o trabalho de Alves e Ussman (2006) que conclui que o principal objetivo desta informação (em particular a gerada pela contabilidade financeira) é o cumprimento das obrigações fiscais. No entanto, esta opinião não é unânime, destacando-se perfis de gestores que defendem que o principal objetivo da contabilidade é fornecer informação para a tomada de decisão. O estudo de Alves (2008), que analisou as decisões dos dirigentes portugueses com recurso à contabilidade, permitiu observar que a IC é importante 
e é utilizada na maioria das decisões. Também Nunes e Serrasqueiro (2004) concluíram que os gestores das pequenas empresas atribuem bastante relevo à IC.

Os estudos referidos validam o recurso à informação quantitativa (no caso em concreto, contabilística) para a tomada de decisão. Contudo, a utilidade da IC para a tomada de decisão pode ser condicionada por diversos aspetos como a tempestividade, a terminologia, as políticas contabilísticas e por diferentes perspetivas entre preparadores e utilizadores (Pierce e O’Dea, 2003). Ultrapassar estas restrições é importante, pois gestores com melhor informação tomam melhores decisões (Mendonza e Bescos, 2001).

A literatura é vasta na análise dos fatores que afetam as tomadas de decisão, nomeadamente da IC. O estudo realizado por İbicioğlu, Kocabycic e Dalğar (2010) sobre a utilização das demonstrações financeiras (DF) durante o processo de decisão em pequenas e médias empresas, revela que os gestores consideram a experiência como o fator mais importante no processo de tomada de decisão. Por outro lado, Nunes e Serrasqueiro (2004) observaram que o nível de formação dos empresários, nas empresas cuja contabilidade é preparada externamente, corresponde ao ensino básico e secundário, donde poderá resultar uma eventual dificuldade em analisar as DF, desconsiderando a IC nas suas decisões. Já Gouveia, Gonçalves e Fernandes (2015) evidenciam, no estudo relativo à utilidade da contabilidade, no âmbito das ME portuguesas, que os gestores utilizam, na sua grande maioria, a IC e que a utilização dessa ferramenta está associada ao setor de atividade, à longevidade da empresa, à dimensão da empresa, medida pelo proxy número de trabalhadores, e à área de formação. Este último aspeto reveste-se de particular interesse, em virtude da atribuição de maior utilidade da IC na tomada de decisão pelos gestores com formação nas áreas económicas.

A idade da empresa (Serrasqueiro e Nunes, 2012), o estilo orçamental (Hopwood, 1972), a estrutura financeira (Branch, 1978), contextos específicos, medidas políticas e institucionais (Guler, 2007) e a estratégia sobre o conhecimento (Knowledge Strategy) (Kasten, 2007), entre outros, são também fatores analisados.

Para além dos fatores referidos, é de considerar, ainda, as diferenças explicadas por traços de personalidade (ex., Mihaela, 2015), estilo cognitivo (ex., Peterson, Rayner e Armstrong, 2009), processamento de informação e heurísticas (ver Barreto, Macedo e Alves 2013), intuição (ver Sadler-Smith e Burke-Smalley, 2015) e estilo de gestão (liderança).

Dada a crescente complexidade e diversidade nas funções dos gestores, aumentou o interesse pelas variáveis biográficas, em particular o género. Impõe-se saber se existirão diferenças de género no comportamento entre os gestores. Adams e Funk (2011) afirmam que existem diferenças ao nível dos principais valores e atitudes de risco. As gestoras são mais benevolentes e universalmente preocupadas, mas menos focadas na realização e no poder que os homens. São também menos focadas nas tradições e na segurança, o que sugere que em car-
Alguns estudos afirmam que existem diferenças ao nível dos principais valores e atitudes de risco. As gestoras são mais benevolentes e universalmente preocupadas, mas menos focadas na realização e no poder que os homens.

Estas conclusões contrariam outos estudos, que afirmam que os responsáveis financeiros femininos são mais adversos ao risco. 
gos diretivos são menos adversas ao risco na tomada de decisão. Estas conclusões contrariam Francis et al. (2014), que afirmam que os responsáveis financeiros femininos são mais adversos ao risco. Também nos estudos de Harris et al. (2006) é referido que as mulheres são mais cautelosas e menos agressivas em diversos contextos de decisões. Um perfil de risco do gestor reflete-se na IC. Gestores mais cautelosos adotam políticas contabilísticas mais conservadoras, o que conduz a que se decida sobre informação de um modo mais prudente. Francis et al. (2014) concluem que as gestoras por serem mais adversas ao risco tendem a adotar políticas de relato mais conservadoras. Nesta ótica de prudência, tendem a criar estimativas de maior qualidade (Barua et al., 2010).

Vários estudos têm procurado avaliar as diferenças de género no que diz respeito aos processos de processamento de informação (ver Meyers-Levy e Loken, 2015), decisão e resolução de problemas (ex., Charness e Gneezy, 2012). A este propósito o estudo de Delaney et al. (2015) com 1075 participantes (56,2\% mulheres) mostrou que as mulheres recorrem menos ao estilo intuitivo. Por seu lado, Filho et al. (2012) observaram uma relação entre o género e a ocorrência de heurísticas em decisões orçamentais.

Apesar de existirem diversos estudos em diferenças de género no desempenho organizacional (ver Gil, Espejo e Gallego, 2010), poucos exploraram a hipótese da importância e da utilidade da IC para a tomada de decisão ser percecionada de forma distinta em função do género. Face ao exposto, assume-se que existem diferenças de género quanto à utilidade atribuída e à importância da IC em função da sua utilização para a tomada de decisão.

\section{Estudo empírico}

O presente estudo é correlacional e considerou dois objetivos: o primeiro pretendeu observar as diferenças de género relativamente à utilização da IC e servir de suporte ao design bifatorial para a prossecução do objetivo principal; e o segundo observar as diferenças de género na utilidade percebida e na importância atribuída à IC.

\section{Hipóteses de investigação}

H1: Existem diferenças entre géneros na utilização da IC na tomada de decisão.

$\mathrm{H} 2$ : Existem diferenças de género na importância e perceção da utilidade da informação contabilística nos não utilizadores.

H3: Existem diferenças de género na importância e perceção da utilidade da informação contabilística nos utilizadores.

\section{Universo e amostra}

O universo é composto pelas ME sob a forma jurídica de sociedade e com atividade não financeira e não seguradora, cuja sede se situa em Portugal continental, e que totalizam 254764 entidades (INE, 2013).

A identificação das entidades a contactar apoiou-se, por conveniência, numa 
base de dados de contactos (link B2B CRM), pelo que a amostra não tem natureza aleatória. Por questões éticas, excluíram-se desta base as empresas com atividades de contabilidade, auditoria e consultoria fiscal (CAE 69200), resultando em 65893 entidades. Obtiveram-se 789 respostas, das quais se validaram 609 (amostra), representando 0,24\% do universo, sendo que 25,5\% dos respondentes são mulheres.

Considerando a variável género, apresenta-se no Quadro 1 valores descritivos agrupados por setor, região, forma jurídica e idade da empresa. No Quadro 2 (p. 44),os dados estão agrupados por características do gestor (idade, habilitações, área de formação e anos de experiência).

\section{Procedimento e instrumento de recolha de dados}

$\mathrm{O}$ instrumento de recolha de dados foi o inquérito, disponibilizado online durante dois meses, tendo sido solicitado aos gestores o seu preenchimento.

$\mathrm{O}$ inquérito estava organizado em seis grupos:

1) Variáveis biográficas dos gestores: género, idade, habilitações académicas e profissionais;

2) Fontes de informação contabilística: local de preparação, pessoal administrativo, subsistemas contabilísticos, periodicidade e quem toma iniciativa de obtenção da informação. Inclui ainda uma questão relativa à utilização da informação contabilística (sim versus não);

Quadro 1 Empresa

\begin{tabular}{|c|l|c|c|c|c|}
\hline \multirow{2}{*}{ Variável } & \multirow{2}{*}{ Subdivisão } & \multicolumn{2}{|c|}{ Mulher } & \multicolumn{2}{c|}{ Homem } \\
\cline { 2 - 6 } & & Frequência & \% & Frequência & $\%$ \\
\hline \multirow{4}{*}{ Setor } & Primário & 2 & 1,3 & 7 & 1,5 \\
\cline { 2 - 6 } & Secundário & 15 & 9,7 & 81 & 17,8 \\
\cline { 2 - 6 } & Terciário & 138 & 89,0 & 366 & 80,6 \\
\hline \multirow{4}{*}{ Região } & Lisboa & 59 & 38,1 & 135 & 29,7 \\
\cline { 2 - 6 } & Norte & 39 & 25,2 & 150 & 33,0 \\
\cline { 2 - 6 } & Centro & 37 & 23,9 & 114 & 25,1 \\
\cline { 2 - 6 } & Algarve & 10 & 6,5 & 35 & 7,7 \\
\cline { 2 - 6 } & Alentejo & 10 & 6,5 & 20 & 4,4 \\
\hline \multirow{4}{*}{$\begin{array}{c}\text { Forma } \\
\text { jurídica }\end{array}$} & Por quotas & 118 & 76,1 & 325 & 71,6 \\
\cline { 2 - 6 } & Unipessoal & 32 & 20,6 & 111 & 24,4 \\
\cline { 2 - 6 } & SA & 4 & 2,6 & 15 & 3,3 \\
\cline { 2 - 6 } & Cooperativa & 1 & 0,6 & 3 & 0,7 \\
\hline \multirow{4}{*}{$\begin{array}{l}\text { Idade da } \\
\text { empresa }\end{array}$} & Entre 1 e 5 & 45 & 29,0 & 143 & 31,5 \\
\cline { 2 - 6 } & 5 e 10 & 41 & 26,5 & 118 & 26,0 \\
\cline { 2 - 6 } & 10 e 20 & 43 & 27,7 & 123 & 27,1 \\
\cline { 2 - 6 } & Mais de 20 & 26 & 16,8 & 70 & 15,4 \\
\hline
\end{tabular}


Quadro 2 Gestor

\begin{tabular}{|c|l|c|c|c|c|}
\hline \multirow{2}{*}{ Variável } & \multirow{2}{*}{ Subdivisão } & \multicolumn{2}{|c|}{ Mulher } & \multicolumn{2}{c|}{ Homem } \\
\cline { 3 - 6 } & Frequência & \% & Frequência & $\%$ \\
\hline \multirow{4}{*}{ Idade } & Entre 18-25 & 3 & 1,9 & 6 & 1,3 \\
\cline { 2 - 6 } & Entre 26-35 & 37 & 23,9 & 97 & 21,4 \\
\cline { 2 - 6 } & Entre 36-45 & 58 & 37,4 & 141 & 31,1 \\
\cline { 2 - 6 } & Entre 46- 55 & 41 & 26,5 & 135 & 29,7 \\
\cline { 2 - 6 } & Mais de 55 & 16 & 10,3 & 75 & 16,5 \\
\hline \multirow{4}{*}{ Habilitações } & Até 9. ${ }^{\circ}$ Ano & 7 & 4,5 & 51 & 11,2 \\
\cline { 2 - 6 } & Secundário & 25 & 16,1 & 122 & 26,9 \\
\cline { 2 - 6 } & Superior & 123 & 79,4 & 281 & 61,9 \\
\hline \multirow{4}{*}{ Área de formação } & Económica & 108 & 69,7 & 307 & 67,6 \\
\cline { 2 - 6 } & Outras & 47 & 30,3 & 147 & 32,4 \\
\hline \multirow{5}{*}{ Anos de experiência } & Entre 1 e 5 & 48 & 31,0 & 97 & 21,4 \\
\cline { 2 - 6 } & 5 e 10 & 42 & 27,1 & 119 & 26,2 \\
\cline { 2 - 6 } & 10 e 20 & 44 & 28,4 & 132 & 29,1 \\
\cline { 2 - 6 } & Mais de 20 & 21 & 13,5 & 106 & 23,3 \\
\hline \hline
\end{tabular}

3) Utilizadores de informação contabilística: frequência (numa escala de 1 - nunca a 5 - mensalmente); tipo de informação contabilística utilizada (balanço, rácios, etc.), grau de concordância em relação a vários aspetos sobre a utilidade da contabilidade - 13 itens numa escala crescente tipo Likert de 1 (discordo totalmente - DT) a 5 (concordo totalmente - CT) - e a importância atribuída à informação contabilística quando toma decisões ( 1 item numa escala crescente tipo Likert de 1 a 10) baseado na medida de Cummins e Gullone (2000);

4) Não utilizadores de informação contabilística: avalia o grau de concordância sobre as várias razões da não utilização da informação contabilística - 14 itens numa escala crescente tipo Likert de 1 (DT) a 5 (CT);

5) Fatores para a tomada de decisão: centrados no gestor e de natureza exógena - 14 itens numa escala crescente tipo Likert de 1 (DT) a 5 (CT);

6) Custo de contexto: avaliação da contabilidade (dicotómica: mais-valia versus custo desnecessário), e peso dos dispêndios suportados pela entidade - 4 itens numa escala crescente tipo Likert de 1 (DT) a 5 (CT). Este grupo inclui uma questão final que pretende aferir se os gestores continuariam a recorrer ao serviço de contabilidade, caso não fosse uma obrigação legal (sim versus não).

Os grupos 1,2, 5 e 6 eram de resposta obrigatória para todos os inquiridos. O grupo 3 era destinado aos inquiridos que tivessem respondido sim no grupo 2 e o grupo 4 para quem respondeu não.

$\mathrm{O}$ inquérito foi sujeito a pré-teste tendo-se procedido ao envio de 10 inqué- 
ritos na versão preliminar a empresas pré-selecionadas, a fim de se identificar problemas nas perguntas que justificassem uma modificação da redação ou alteração do formato. O pré-teste validou a clareza e compreensibilidade das questões, dando viabilidade ao envio do questionário na versão final.

\section{Tratamento de dados}

Recorreu-se a estatísticas descritivas e a testes não paramétricos (em virtude de a amostra ser não aleatória e da não normalidade da distribuição dos dados das variáveis). Para o teste de independência das variáveis nominais realizou-se o teste qui-quadrado $\left(\chi^{2}\right)$. Considerou-se um $p<0,05$, se bem que sejam apresentados alguns resultados $\operatorname{com} p<0,1$.

As variáveis foram agrupadas em três vetores - estrutural, empresa e gestor - fundamentadas em trabalhos com natureza análoga ou de natureza exploratória e testadas sobre a sua relação com o género. $\mathrm{O}$ vetor relacionado com o contexto da empresa foi denominado como «estrutural». O vetor «empresa» inclui diversos indicadores económico-financeiros relativos à sociedade. E o vetor relacionado com o perfil do gestor designa-se «gestor».

Em suma, quanto aos três vetores, a abrangência é a seguinte:

- Estrutural: região, setor, forma jurídica, idade da empresa (Alves, 2008; Gouveia et al., 2015; Lucena et al., 2011; Serrasqueiro e Nunes, 2012);

- Empresa: volume de negócios, número de trabalhadores, total ativo, resultados do período, capital próprio, return on assets, return on equity, liquidez geral, endividamento e solvabilidade (Gouveia et al., 2015; Lucena et al., 2011);

- Gestor: idade, habilitações, área de formação e anos de experiência (Gouveia et al., 2015; İbicioğlu et al., 2010; Lucena et al., 2011; Taylor, 1975).

\section{Resultados}

\section{Análise descritiva}

Do agrupamento da amostra por setores de atividades, resultou uma distribuição setorial próxima à do universo. Verifica-se também uma forte aproximação entre a amostra e os dados nacionais, quer em relação à distribuição por regiões, quer quanto à forma jurídica.

Os resultados no que concerne às percentagens de gestoras são consistentes, quer com INE (2013), quer com a Informa D\&B (2012). No que se refere às variáveis no âmbito «estrutural», as frequências de respostas são relativamente semelhantes nos dois géneros, observando-se um maior número de respostas de empresas geridas por mulheres no setor dos serviços e de empresas sediadas em Lisboa. Neste enquadramento, verifica-se que a idade da empresa gerida por cada um dos géneros é percentualmente semelhante, o que não coincide com os dados da Informa D\&B (2012).

No vetor gestor, verifica-se, à semelhança do que foi anteriormente assinalado, que a mulher gestora é mais jovem e com mais habilitações $(p<0,05)$ $63,2 \%$ tem menos de 45 anos e 79,4\% detêm um curso superior. No caso dos 
gestores masculinos 53,7\% têm até 45 anos e 61,9\% têm formação superior. Em termos de experiência, 52,4\% dos homens gerem empresas há mais de 10 anos, por oposição aos $41,9 \%$ das mulheres para o mesmo período de tempo ( $p<$ $0,05)$.

A área de formação foi agregada em dois grupos: formação económica (economia, finanças, auditoria, contabilidade, gestão) versus outras formações (engenharia, comerciais e outras sem formação específica). Não há diferenças estatisticamente significativas entre os grupos de formação, atendendo ao género.

No que concerne às aplicações contabilísticas utilizadas, verificou-se uma relação significativa $(p<0,05)$ entre os géneros na utilização da contabilidade orçamental. Os homens utilizam mais esta aplicação contabilística dentro da própria empresa. As mulheres recorrem mais ao outsourcing para a mesma.

\section{Teste de hipóteses}

Atendendo ao âmbito do estudo, constituiram-se dois grupos baseados no género. Inicialmente cada grupo foi subdividido em dois: utilizadores e não utilizadores.

Para H1 concluiu-se não existir evidência estatística que indique que a variável género é fator determinante para a utilização, ou não, da $\operatorname{IC}\left(\chi^{2}=0,275\right.$; $p=0,600)$.

No grupo dos que utilizam a contabilidade não se evidenciou qualquer relação significativa.

Para testar H2, do teste de confiabilidade resultou um alfa de Cronbach de 0,688 (aceitável). No Quadro 3 referem-se as relações estatisticamente significativas, tendo por base a variável género, as taxas de respostas obtidas nas questões de escala de 5 pontos e o resultado do teste de qui-quadrado realizado entre a variável género e cada uma dessas questões.

Verifica-se evidência estatística relativa à diferença da perceção dos fatores explicativos para a não utilização da contabilidade, em função do género, validando parcialmente a $\mathrm{H} 2$.

A não necessidade da IC para tomar decisões é apontada por $50,6 \%$ dos homens (versus $31,3 \%$ para as mulheres). A razão da não utilização por falta da entrega atempada da IC é apontada por $53,1 \%$ das mulheres (versus $27,1 \%$ nos homens). É evidente o resultado do forte impacto da carga tributária na consciência dos gestores. Nesse aspeto, esse efeito é menos valorizado pelas mulheres $(71,9 \%$, versus $83,5 \%)$ visto que discordam mais sobre se a IC é essencialmente um meio para apurar impostos. As mulheres reconhecem, mais que os homens $(93,8 \%$ versus $80 \%)$, que para compreender a IC é importante ter conhecimentos em contabilidade de gestão e finanças, observando-se que em $94 \%$ das mulheres que concordam com essa afirmação $62,5 \%$ concordam totalmente. Dos $80 \%$ dos homens que concordam apenas $28 \%$ são totalmente concordantes. No mesmo sentido, segue a opinião de

\section{O estudo verificou} que a mulher gestora é mais jovem e com mais habilitações $(p<0,05)-63,2 \%$ tem menos de 45 anos e $79,4 \%$ detêm um curso superior. No caso dos gestores masculinos $53,7 \%$ têm até 45 anos e $61,9 \%$ têm formação superior. Em termos de experiência, $52,4 \%$ dos homens gerem empresas há mais de 10 anos, por oposição aos $41,9 \%$ das mulheres para o mesmo período de tempo $(p<0,05)$. 
Quadro 3 Fatores da utilidade percebida da contabilidade

\begin{tabular}{|l|c|c|c|c|c|}
\hline \multirow{2}{*}{ Variável } & \multicolumn{2}{|c|}{ Discordo* } & \multicolumn{2}{c|}{ Concordo** } & Chi-Square Tests \\
\cline { 2 - 6 } & Mulher & Homem & Mulher & Homem & Sig. (2-sided) \\
\hline $\begin{array}{l}\text { Não necessito da informação } \\
\text { contabilística para tomar } \\
\text { decisões na empresa }\end{array}$ & $53,1 \%$ & $34,1 \%$ & $31,3 \%$ & $50,6 \%$ & 0,033 \\
\hline $\begin{array}{l}\text { A informação contabilística } \\
\text { não é entregue em tempo útil }\end{array}$ & $37,5 \%$ & $48,2 \%$ & $53,1 \%$ & $27,1 \%$ & 0,061 \\
\hline $\begin{array}{l}\text { A informação contabilística é } \\
\text { essencialmente um meio para } \\
\text { apurar impostos para o Estado }\end{array}$ & $21,9 \%$ & $10,6 \%$ & $71,9 \%$ & $83,5 \%$ & 0,089 \\
\hline $\begin{array}{l}\text { Para compreender melhor a } \\
\text { informação, é importante ter } \\
\text { alguns conhecimentos em } \\
\text { contabilidade de gestão e } \\
\text { finanças }\end{array}$ & $6,3 \%$ & $10,6 \%$ & $93,8 \%$ & $80,0 \%$ & 0,012 \\
\hline $\begin{array}{l}\text { Com conhecimentos em } \\
\text { contabilidade de gestão e } \\
\text { finanças seria possível obter } \\
\text { melhores resultados na } \\
\text { empresa }\end{array}$ & $12,5 \%$ & $28,2 \%$ & $68,8 \%$ & $55,3 \%$ & 0,022 \\
\hline $\begin{array}{l}\text { N= 117; Resposta neutra não considerada. *Resposta discordo totalmente e discordo em parte; **Resposta concordo } \\
\text { totalmente e concordo em parte }\end{array}$ & & & & \\
\hline
\end{tabular}

que com conhecimentos nestas áreas seria possível obter melhores resultados, verificando-se que estão de acordo com esta ideia $69 \%$ das mulheres face a $55 \%$ dos homens.

Quanto ao efeito dos fatores pessoais, internos ou externos, na tomada de decisão, destaca-se no Quadro 4 os resultados estatisticamente significativos de acordo com o género, validando a $\mathrm{H} 3$. No conjunto dos itens relativos aos fatores pessoais, internos ou externos obteve-se um alfa de Cronbach aceitável (0,661 e 0,734, respetivamente). Tal como no Quadro 3, divulgam-se as percentagens em termos de importância para cada item e o resultado do teste de qui-quadrado, em função do género.

Numa perspetiva associada aos indivíduos, observa-se que é considerado importante o grau de formação do gestor na tomada de decisão, dando a mulher $(87,7 \%)$ ligeiramente mais importância a esta qualidade do que os homens $(80,4 \%)$. Quanto à intuição como fator decisivo na tomada de decisão, os resultados são bastante semelhantes, sendo que o homem atribui mais importância a essa característica no momento de decidir. Evidencia-se a influência dos fatores políticos e económicos na tomada de decisão, sem grandes divergências entre géneros. Constata-se a preponderância significativa das mulheres (97,4\% versus $89,9 \%$ ) na opinião de que os resultados da empresa exercem influência na tomada de decisão. 


\begin{tabular}{|l|c|c|c|c|c|}
\hline \multicolumn{1}{|c|}{ Variável } & \multicolumn{2}{|c|}{$\begin{array}{c}\text { Pouco ou nada } \\
\text { importante }\end{array}$} & \multicolumn{2}{|c|}{$\begin{array}{c}\text { Importante ou } \\
\text { muito importante }\end{array}$} & $\begin{array}{c}\text { Chi-Square } \\
\text { Tests } \\
\text { Sig. (2-sided) }\end{array}$ \\
\cline { 2 - 5 } & Mulher & Homem & Mulher & Homem & \\
\hline $\begin{array}{l}\text { Grau de importância da } \\
\text { formação pessoal e } \\
\text { académica na tomada de } \\
\text { decisão }\end{array}$ & $1,3 \%$ & $2,6 \%$ & $87,7 \%$ & $80,4 \%$ & 0,049 \\
\hline $\begin{array}{l}\text { Grau de importância da } \\
\text { intuição na tomada de } \\
\text { decisão }\end{array}$ & $17,4 \%$ & $17,4 \%$ & $43,9 \%$ & $49,3 \%$ & 0,062 \\
\hline $\begin{array}{l}\text { Influência dos fatores } \\
\text { económicos na tomada de } \\
\text { decisão }\end{array}$ & $0,6 \%$ & $2,9 \%$ & $93,5 \%$ & $89,2 \%$ & 0,075 \\
\hline $\begin{array}{l}\text { Influência dos fatores } \\
\text { políticos na tomada de } \\
\text { decisão }\end{array}$ & $31,6 \%$ & $33,3 \%$ & $38,7 \%$ & $31,9 \%$ & 0,095 \\
\hline $\begin{array}{l}\text { Influência dos resultados } \\
\text { da empresa na tomada de } \\
\text { decisão }\end{array}$ & $0,6 \%$ & $2,0 \%$ & $97,4 \%$ & $89,9 \%$ & 0,004 \\
\hline
\end{tabular}

Resposta neutra não considerada

Quadro 5 Custo da contabilidade

\begin{tabular}{|l|c|c|c|c|c|}
\hline \multirow{2}{*}{ Variável } & \multicolumn{2}{|c|}{ Discordo* } & \multicolumn{2}{c|}{ Concordo** } & $\begin{array}{c}\text { Chi-Square } \\
\text { Tests }\end{array}$ \\
\cline { 2 - 6 } & Mulher & Homem & Mulher & Homem & Sig. (2-sided) \\
\hline $\begin{array}{l}\text { Prefiro pagar mais pelo } \\
\text { serviço de contabilidade } \\
\text { para ter uma informação de } \\
\text { confiança e qualidade }\end{array}$ & $9,0 \%$ & $11,7 \%$ & $82,6 \%$ & $69,2 \%$ & 0,017 \\
\hline $\begin{array}{l}\text { Já deixei de ter ou fazer } \\
\text { algo importante para a } \\
\text { empresa, para poder } \\
\text { cumprir com os } \\
\text { pagamentos pelo serviço de } \\
\text { contabilidade }\end{array}$ & $56,1 \%$ & $52,4 \%$ & $25,2 \%$ & $21,8 \%$ & 0,010 \\
\hline $\begin{array}{l}\text { Resposta neutra não considerada; *Resposta discordo totalmente e discordo em parte; } \\
\text { **Resposta concordo totalmente concordo em parte }\end{array}$ & & & & \\
\hline
\end{tabular}

procuram determinar a importância da contabilidade na ótica do custo, apresentando-se, em percentagens, o nível de concordância de cada item e o resultado do teste de qui-quadrado. 
Quadro 6 Dados financeiros - Rácio endividamento

\begin{tabular}{|l|c|c|c|}
\hline \multirow{2}{*}{ Género } & \multicolumn{3}{|c|}{ Rácio endividamento } \\
\cline { 2 - 4 } & {$[\mathbf{0} \%)$} \\
\hline Mulher & $32,5 \%$ & {$[\mathbf{5 0} ; \mathbf{1 0 0}]$} & $>\mathbf{1 0 0}$ \\
\hline Homem & $34,3 \%$ & $42,2 \%$ & $25,3 \%$ \\
\hline
\end{tabular}

Quadro 7 Dados financeiros - Resultados líquidos do período

\begin{tabular}{|l|c|c|c|}
\hline \multirow{2}{*}{ Género } & \multicolumn{3}{|c|}{ Resultado líquido do período } \\
\cline { 2 - 4 } & $\mathbf{<}$ & {$[\mathbf{0} ; \mathbf{2 5} \mathbf{0 0 0}]$} & $>\mathbf{2 5} \mathbf{0 0 0}$ \\
\hline Mulher & $49,0 \%$ & $43,2 \%$ & $7,7 \%$ \\
\hline Homem & $38,8 \%$ & $52,4 \%$ & $8,8 \%$ \\
\hline
\end{tabular}

Constata-se que 82,6\% das mulheres concordam que é preferível pagar mais pelo serviço de contabilidade, para ter uma informação de confiança e qualidade (somente 69,2\% dos homens concordam com essa opção). Quando ocorrem dificuldades financeiras, o que, na hora de decidir, pode obrigar a escolher entre satisfazer um compromisso com um fornecedor ou com outro, o prestador do serviço de contabilidade pode sair prejudicado. Embora a maioria dos gestores não deixem de pagar o serviço de contabilidade em detrimento de outro fornecedor, um quarto das mulheres afirma privilegiar nestas situações os outros fornecedores.

Numa última análise, procedeu-se ao cruzamento entre variáveis do vetor empresa com a variável género. Obtiveram-se dois resultados com nível de significância dentro do parâmetro admitido neste estudo, nomeadamente um $p$ de 0,036 (ver Quadro 6), que evidencia uma relação entre o género e o endividamento da empresa e um $p$ de 0,08 (ver Quadro 7), que associa o género ao resultado da empresa.

Os Quadros 6 e 7 apresentam as frequências obtidas em cada intervalo de cada variável. Evidencia-se a existência de uma maior percentagem de empresas geridas por mulheres com um resultado líquido do período inferior a zero e que uma em cada quatro empresas lideradas por gestoras tem um grau de endividamento acima dos $100 \%$.

\section{Conclusão}

O aumento da diversidade nas organizações tem contribuído para o interesse no impacto que as variáveis biográficas e culturais têm nas organizações, nos processos de trabalho e no desempenho (ex., Lu et al., 2015), em específico, o efeito do género nas funções de gestão. São escassos os trabalhos que estudam a temática do género dos gestores de empresas portuguesas, sobretudo nas de 
menor dimensão. A partir do trabalho de Gouveia et al. (2015) pretendeu-se aprofundá-lo na perspetiva do género, em particular as diferenças de género na utilização da contabilidade, como fonte de informação para a tomada de decisão nas microempresas.

Apesar de existirem hoje em dia mais mulheres em lugares de gestão, este estudo é consentâneo com as diferenças percentuais em relação aos homens e às características biográficas dos gestores (tempo de experiência, formação superior e idade). Em conformidade com os dados divulgados pelos relatórios da Comissão Europeia, do INE e da Informa D\&B, o estudo mostra uma reduzida percentagem de mulheres à frente das empresas, que são mais jovens, possuem mais habilitações, mas detêm menos anos de experiência em gestão quando comparado com os seus congéneres masculinos. De acordo com a literatura, este fator é apontado como produzindo um efeito na performance das empresas, dado a sua menor prática (ex., Ahern e Dittmar, 2012).

À semelhança de Pereira (2013), também neste estudo se verificou que existe uma maior percentagem de mulheres a gerir empresas com pior desempenho económico-financeiro. $\mathrm{O}$ significado destes resultados não deve ser interpretado individualmente. A leitura deve ser feita no cômputo geral tendo em consideração todos os fatores envolventes. Verifica-se que existe uma clara desvantagem das mulheres quanto ao tempo que exercem estas funções. Assumidamente as mulheres detêm mais habilitações, mas os conhecimentos teóricos não substituem inteiramente a prática adquirida ao longo dos muitos anos de experiência. Essa limitação poder-se-á tornar desvantajosa para as gestoras e as suas empresas, mas certamente, com o decorrer do tempo será um problema que tenderá a desaparecer.

Não se encontrou evidência estatística, com base no género, no grupo de utilizadores da IC para a tomada de decisão.

Para os não utilizadores, destaca-se diferenças entre homens e mulheres em algumas vertentes designadamente sobre a necessidade da IC para a tomada de decisões, onde as mulheres manifestam maior concordância. Apesar desta concordância, trata-se de não utilizadores da contabilidade. Esse facto deve-se a dois fatores: admitirem dificuldades em compreender a informação e acesso à informação em tempo útil. Neste sentido, as mulheres atribuem mais importância aos conhecimentos necessários nas áreas da contabilidade e afins. Assim, as mulheres parecem valorizar mais do que os homens a formação pessoal e académica na tomada de decisão.

Estes resultados poderão, por um lado, estar associados à perceção de menores conhecimentos comparados com os homens e não a diferenças reais, traduzindo a avaliação de competências expressas nos estereótipos de género afetuosas, dependentes, menos competentes em matemática (ver Gonçalves et al., 2015) - com as consequências que implica. Dito de outra forma, os estereótipos condicionam os alvos de estereótipo (neste caso, as mulheres) levando-os a comportar-se de acordo com o estereótipo através de processos de confirmação comportamental (ex., Snyder, 1984), autorrealização de profecias

\section{Assumidamente} as mulheres detêm mais habilitações, mas os conhecimentos teóricos não substituem inteiramente a prática adquirida ao longo dos muitos anos de experiência. Essa limitação poder-se-á tornar desvantajosa para as gestoras e as suas empresas, mas certamente, com o decorrer do tempo será um problema que tenderá a desaparecer. 
(Merton, 1948) ou de ameaça de estereótipo (ex. Logel et al., 2009; Maass, D’Ettole e Cadinu, 2008; McGlone, Aronson e Kobrynowicz., 2006; Steele e Aronson, 1995).

Por outro lado, o facto de as mulheres apresentarem estilos diferentes de processo de decisão também pode suportar a perceção de menores conhecimentos e/ou menos confiança em si. A este propósito, o facto de as mulheres preferirem pagar mais por um melhor serviço de contabilidade, interpreta-se, como sendo sinal de um perfil mais cauteloso, primando por uma informação segura e com qualidade, o que é coincidente com Harris et al. (2006) e Barua et al. (2010) e com um estilo de decisão mais dependente (Delaney et al., 2015).

Estudos futuros poderão contribuir para avaliar o efeito que estas variáveis, experiência, estereótipos, estilos de decisão ou outras variáveis distintivas dos géneros (ver Meyers-Levy e Loken, 2015) têm na avaliação do uso da informação contabilística. Contrariamente a Filho et al. (2012), relativamente à intuição como fator decisivo na tomada de decisão, apesar de os homens atribuírem mais valor a essa característica, os resultados entre géneros são bastante semelhantes. A diferença encontrada entre estes estudos pode estar associada ao facto de as mulheres utilizarem mais o outsourcing, pelo que a informação contabilística utilizada (em particular no que se refere aos outputs gerados pela contabilidade orçamental) é mais isenta de juízos de valor do gestor associados à sua preparação e apresentação.

A principal limitação a assinalar neste trabalho prende-se com o carácter não aleatório da amostra que restringe a generalização dos resultados. Contudo, existem alguns fatores que contribuem para a sua representatividade, nomeadamente a heterogeneidade, dado que se obtiveram respostas provenientes de 19 tipos de atividades, de entre as 22 presentes na tabela CAE (Classificação de Atividades Económicas).

Relativamente ao instrumento de recolha, as respostas poderão estar condicionadas ao efeito de desejabilidade social devido ao carácter das questões. Por outro lado, a utilização de inquéritos online não permite garantir quem respondeu. Controlou-se esta incerteza através do contacto telefónico a diversas entidades selecionadas aleatoriamente.

Existe a necessidade de continuar a elaborar mais trabalhos concernentes ao género, em diferentes contextos, de forma a entender melhor a realidade do país. Revelar-se-ia útil conhecer também os resultados provenientes da gestão de diferentes mix de géneros ( $\mathrm{HH}, \mathrm{HM}, \mathrm{MM}$ ). Atendendo que os comportamentos e resultados dos mesmos afetam a mudança de atitudes, seria importante considerar futuramente estudos transversais, que analisem ao longo do tempo os processos de tomada de decisão dos gestores e a relação com a importância, a utilidade percebida e a utilização da IC e com o desempenho da empresa.

Considera-se que o presente estudo contribui para a literatura na medida em que preenche uma lacuna existente em estudos nacionais no que diz respeito à

\section{As presentes}

conclusões poderão

ser úteis em sede

das opções de política

governamental, no que

concerne a medidas

que fomentem

a igualdade de género. 
contabilidade e ao género. Neste sentido, as presentes conclusões poderão ser úteis em sede das opções de política governamental, no que concerne a medidas que fomentem a igualdade de género.

Nota: Este artigo foi financiado por fundos provenientes da Fundação para a Ciência e Tecnologia, através do projecto UID/SAC/4020/2013.

\section{Referências bibliográficas}

ADAMS, R. e FUNK, P. (2011), «Beyond the glass ceiling: does gender matter?». Management Science, vol. 58(2), pp. 219-235.

AHERN, K. e DITTMAR, A. (2012), «The changing of the boards: the impact on firm valuation of mandated female board representation». Quartely Journal of Economics, vol. 127(1), pp. 137-197.

AJZEN, I. e FISHBEIN, M. (1980), Understanding Attitudes and Predicting Social Behavior. Englewood-Cliffs, NJ: Prentice-Hall.

ALVES, M. (2008), «A contabilidade nas decisões de gestão dos dirigentes Portugueses». Revista Universo Contábil, vol. 4(3), pp. 124-137.

ALVES, M. e USSMAN, A. (2006), «O valor da informação contabilística na óptica do produtor e do utilizador da informação - estudo empírico». Revista de Contabilidade e Gestão, vol. 2, pp. 123-142.

BAIOCCO, R.; LAGHI, F. e D’ ALLESSIO, M. (2009), «Decision-making style among adolescents: Relationship with sensation seeking and locus of control». Journal of Adolescence, vol. 32(4), pp. 963-976.

BARRETO, P.; MACEDO, M. e ALVES, F. (2013), «Tomada de decisão e teoria dos prospectos em ambiente contábil: uma análise com foco no efeito framing». Revista de Gestão, Finanças e Contabilidade, vol. 3(2), pp. 61-79.

BARUA, A.; DAVIDSON, L.; RAMA, D. e THIRUVADI, S. (2010), «CFO Gender and accruals quality». Accounting Horizons, vol. 24(1), pp. 25-39.

BRANCH, B. (1978), «The impact of operating decisions and ROI dynamics». Financial Management, vol. 7(4), pp. 54-60.

CAPLAN, E.H. (1966), «Behavioral assumptions of management accounting». The Accounting Review, vol. 41, n. ${ }^{\circ}$ 3, pp. 496-509.

CHARNESS, G. e GNEEZY, U. (2012), «Strong evidence for gender differences in risk taking». Journal of Economic Behavior \& Organization, vol. 83(1), pp. 50-58.

CHEN, C.; DING, Y. e XU, B. (2014), «Convergence of accounting standards and foreign direct investment». The International Journal of Accounting, vol. 49(1), pp. 53-86.

COMISSÃO EUROPEIA (2003), Recomendação da Comissão de 6 de maio de 2003 relativa à definição de micro, pequenas e médias empresas, n. ${ }^{\circ}$ 2003/361/CE. 20 de Maio de 2003. http://eur-lex.europa.eu/LexUriServ/LexUriServ.do?uri=OJ:L:2003:124:0036:0041:PT:PDF.

CUMMINS, R. e GULLONE, E. (2000), «Why we should not use 5-point Likert scales: the case for subjective quality of life measurement». Second International Conference on Quality of Life in Cities, Singapore.

DAVENPORT, T. (2009), «Make better decisions». Harvard Business Review, November, pp. 1-7.

DAVIDSON, H.J. e TRUEBLOOD, R.M. (1961), «Accounting for decision-making». TheAccounting Review, vol. 36, n. ${ }^{\circ}$, pp. 577-582.

DELANEY, R.; STROUGH, J.; PARKER, A. e DE BRUIN, W. (2015), «Variations in decision-making profiles by age and gender: A cluster-analytic approach». Personality and Individual Differences, vol. 85, pp. 19-24,

EUROPEAN INSTITUTE FOR GENDER EQUALITY (2013), «Gender Equality Index: 
Country Profiles». Itália. 13 de junho de 2013. http://eige.europa.eu/content/gender-equality-index.

FERREIRA, C. (2013), «Influência do género na contabilidade». XIV Congresso Internacional de Contabilidade e Auditoria, Lisboa, outubro.

FILHO, R.; BRUNI, A. e SAMPAIO, M. (2012), «A influência do gênero, idade e formação na presença de heurísticas em decisões de orçamento: um estudo quase experimental». Revista Universo Contábil, vol. 8(2), pp. 103-117.

FRANCIS, B.; HASAN, I.; PARK, J. e WU, Q. (2014), «Gender differences in financial reporting decision-making: evidence from accounting conservatism». Bank of Finland. FinlandResearch Discussion Papers. http://www.suomenpankki.fi/pdf/172914.pdf.

GIL, D.; ESPEJO, C. e GALLEGO, A. (2010), «The relationship between diversity, gender and accounting». Revista de Contabilidad-Spanish, vol. 13(1), pp. 9-16.

GONÇALVES, G.; ORGAMBÍDEZ-RAMOS, A.; GIGER, J.; SANTOS, J. e GOMES, A. (2015), «Evidencias de validez de la adaptación portuguesa de la escala de sexismo ambivalente». Revista de Psicología Social, vol. 30(1), pp. 152-181.

GOUVEIA, H.; GONÇALVES, C. e FERNANDES, J. (2015), «A utilidade da contabilidade para os gestores das microempresas ». Revista de Contabilidade e Gestão, vol. 16, pp. 77 $-104$.

GULER, I. (2007), «Throwing good money after bad? Political and institutional influences on sequential decision making in the venture capital industry». Administrative Science Quarterly, vol. 52(2), 248-285.

HARRIS, C.; JENKINS, M. e GLASER, D. (2006), «Gender differences in risk assessment: Why do women take fewer risks than men?». Judgment and Decision Making, vol. 1(1), pp. 48$-63$.

HOPWOOD, A. (1972), «An empirical study of the role of accounting data in performance evaluation". Journal of Accounting Research, Empirical Research in Accounting: Selected Studies, vol. 10, pp. 156-182.

İBICIOǦLU, H.; KOCABIYIC, T. e DALĞAR, H. (2010), «Financial statement utilization during decision making process in SMEs: A comparative study on European and Turkish managers $\gg$. I.I.B.F Dergisi, vol. XXVIII, pp. 209-226.

INFORMA D\&B (2012), «Perfil da presença feminina no tecido empresarial português ». Março.http://www.informadb.pt/biblioteca/ficheiros/4_Presenca_Feminina_tecido_empresarial_portugues_edicao2.pdf.

INSTITUTO NACIONAL DE ESTATÍSTICA (2013), «Trabalhar no feminino». Março. https://www.ine.pt/xportal/xmain?xpid=INE\&xpgid=inedestaques\&DESTAQUESdest_boui $=154532260 \&$ DESTAQUESmodo $=2$.

KANG, J.-K. e STULZ, R.M. (1997), «Why is there a home bias? An analysis of foreign portfolio equity ownership in Japan». Journal of Financial Economics, vol. 46, n. ${ }^{0}$ 1, pp. 3-28.

KASTEN, J. (2007), «Knowledge strategy and its influence on knowledge organization». Proceedings of the North American Symposium on Knowledge Organization, vol. 1, pp. 44-54 .

LOGEL, C.; WALTON, G.M.; SPENCER, S.J.; ISERMAN, E.C.; VON HIPPEL, W. e BELL, A.E. (2009), «Interacting with sexist men triggers social identity threat among female engineers $\gg$. Journal of Personality and Social Psychology, 96, pp. 1089-1103.

LU, C.-M.; CHEN, S.-J.; HUANG, P.-C. e CHIEN, J.-C. (2015), «Effect of diversity on human resource management and organizational performance». Journal of Business Research, vol. 68(4), pp. 857-861.

LUCENA, W.; VASCONCELOS, M. e MARCELINO, G. (2011), «A evidenciação das informações contábeis geradas pelas micro e pequenas empresas no processo decisório: um estudo no setor de Confecções». REUNIR - Revista de Administração, Contabilidade e Sustentabilidade, vol. 1(1), pp. 35-51. 
MALA, R. e CHAND, P. (2015), «Judgment and decision-making research in auditing and accounting: future research implications of person, task, and environment perspective». Accounting Perspectives, vol. 14(1), pp. 1-50.

MARTIN, A. (1971), «An empirical test of the relevance of accounting information for investment decisions $\gg$. Journal of Accounting Research (Empirical Research in Accounting: Selected Studies), vol. 9, pp. 1-31.

MAASS, A.; D’ETTOLE, C. e CADINU, M. (2008), «Checkmate? The role of gender stereotypes in the ultimate intellectual sport». European Journal of Social Psychology, 38, pp. 231-245 .

MCGLONE, M.S.; ARONSON, J. e KOBRYNOWICZ, D. (2006), «Stereotype threat and the gender gap in political knowledge». Psychology of Women Quarterly, 30, pp. 392-398.

MENDONZA, C. e BESCOS, P. (2001), «An explanatory model of managers' information needs: implications for management accounting». The European Accounting Review, vol. 10(2), pp. 257-289.

MERTON, R.K. (1948), «The self-fulfilling prophecy». Antioch Review, vol. 8, pp. 193-210.

MEYERS-LEVY, J. e LOKEN, B. (2015), «Revisiting gender differences: what we know and what lies ahead». Journal of Consumer Psychology, vol. 25(1), pp. 129-149.

MIHAELA, P. (2015), «Personality variables in decision-making». Procedia: Social and Behavioral Sciences, vol. 187, pp. 658-662.

NUNES, L. e SERRASQUEIRO, Z. (2004), «A informação contabilística nas decisões financeiras das pequenas empresas». Revista Contabilidade \& Finanças, vol. 36, 87-96.

OKOH, L. e UZOKA, P. (2012), «The role of accounting information in the survival of small scale businesses in Warri, Delta State, Nigeria». International Journal of Economic Development Research and Investment, vol. 3(1), pp. 40-44.

PEREIRA, S. (2013), «Diversidade de Género nos Órgãos Sociais das Empresas Portuguesas». Dissertação de Mestrado em Contabilidade e Controlo de Gestão, Faculdade de Economia da Universidade do Porto.

PETERSON, E.; RAYNER, S. e ARMSTRONG, S. (2009), «Researching the psychology of cognitive style and learning style: is there really a future?». Learning and Individual Differences, vol. 19(4), pp. 518-523.

PIERCE, B. e O'DEA, T. (2003), «Management accounting information and the needs of managers: perceptions of managers and accountants compared». The British Accounting Review, vol. 3(35), pp. 257-290.

PORTES, R. e REY, H. (2005), «The determinants of cross-border equity flows». Journal of International Economics, vol. 65(2), pp. 269-296.

ROSSI, S. e VOLPIN, P. (2004), «Cross-country determinants of mergers and acquisitions». Journal of Financial Economics, vol. 74(2), pp. 277-304.

RÜZBAR, N. e KURT, M. (2013), «Manager's roles and profiles: Evidence from Bursa». Procedia - Social and Behavioral Sciences, vol. 99, pp. 240-246.

SADLER-SMITH, E. e BURKE-SMALLEY, L. (2015), «What do we really understand about how managers make important decisions?». Organizational Dynamics, vol. 44(1), pp. 9-16.

SERRASQUEIRO, Z. e NUNES, P. (2012), «Is age a determinant of SMEs' financing decisions? Empirical evidence using panel data models $\gg$. Entrepreneurship Theory and Practice, vol. 36(4), 627-654.

SNYDER, M. (1984), «When belief creates reality». Advances in Experimental Social Psychology, L. Berkowitz (Ed.), vol. 18, pp. 248-306.

SPICER, D. e SADLER-SMITH, E. (2005), «An examination of the general decision-making style questionnaire in two UK sample». Journal of Managerial Psychology, vol. 20(2), pp. 137-149.

STEELE, C.M. e ARONSON, J. (1995), «Stereotype threat and the intellectual test performance of African Americans». Journal of Personality and Social Psychology, 69, pp. 697-711. 
55| Revista de GESTÃO dos Países de Língua Portuguesa

TAYLOR, R.N. (1975), «Age and experience as determinants of managerial information processing and decision-making performance ». Academy of Management Journal, vol. 18, n. ${ }^{\circ}$, pp. 74-81.

ZHANG, G. (2000), «Accounting information, capital investment decisions, and equity valuation: theory and empirical implications ». Journal of Accounting Research, vol. 38(2), pp. 271-295. 